\title{
Navy Bean Powder
}

National Cancer Institute

\section{Source}

National Cancer Institute. Navy Bean Powder. NCI Thesaurus. Code C111687.

The powder form of the cooked navy bean with potential antioxidant and

chemopreventive activities. Navy beans are rich in fiber, minerals, vitamins, and phytochemicals such as flavonoids and phytosterols. They appear to prevent carcinogenesis by inducing tumor cell apoptosis. Intake of navy bean powder may have a beneficial effect on intestinal microflora. 